\title{
A NOTE ON THE TRANSITIVE HURWITZ ACTION ON DECOMPOSITIONS OF PARABOLIC COXETER ELEMENTS
}

\author{
BARBARA BAUMEISTER, MATTHEW DYER, CHRISTIAN STUMP, \\ AND PATRICK WEGENER
}

(Communicated by Patricia L. Hersh)

\begin{abstract}
In this note, we provide a short and self-contained proof that the braid group on $n$ strands acts transitively on the set of reduced factorizations of a Coxeter element in a Coxeter group of finite rank $n$ into products of reflections. We moreover use the same argument to also show that all factorizations of an element in a parabolic subgroup of $W$ also lie in this parabolic subgroup.
\end{abstract}

\section{INTRODUCTION}

Let $(W, T)$ be a dual Coxeter system of finite rank $m$ in the sense of Bes03. This is to say that there is a subset $S \subseteq T$ with $|S|=m$ such that $(W, S)$ is a Coxeter system, and $T=\left\{w s w^{-1}: w \in W, s \in S\right\}$ is the set of reflections for the Coxeter system $(W, S)$. We then call $(W, S)$ a simple system for $(W, T)$ and $S$ a set of simple reflections. Such simple systems for $(W, T)$ were studied by several authors, see e.g. FHM06] and the references therein. In particular, if $S$ is a simple system for $(W, T)$ then so is $w S w^{-1}$ for any $w \in W$. It is moreover shown in FHM06 that for important classes, all simple systems for $(W, T)$ are conjugate to one another in this sense.

A reflection subgroup $W^{\prime}$ is a subgroup of $W$ generated by reflections. It is well known that $\left(W^{\prime}, W^{\prime} \cap T\right)$ is again a dual Coxeter system, see e.g. Dye90. For $w \in W$, a reduced $T$-factorization of $w$ is a shortest length factorization of $w$ into reflections, and we denote by $\operatorname{Red}_{T}(w)$ the set of all such reduced $T$-factorizations. Similarly for a given simple system $(W, S)$, a reduced $S$-factorization of $w$ is a shortest length factorization of $w$ into simple reflections. An element $c \in W$ is called a parabolic Coxeter element for $(W, T)$ if there is a simple system $S=\left\{s_{1}, \ldots, s_{m}\right\}$ such that $c=s_{1} \cdots s_{n}$ for some $n \leq m$. We call the reflection subgroup generated by $\left\{s_{1}, \ldots, s_{n}\right\}$ a parabolic subgroup. The element $c$ is moreover called a standard parabolic Coxeter element for the Coxeter system $(W, S)$.

Remark 1.1. Observe that this definition of parabolic Coxeter elements and parabolic subgroups is more general than usual. The simplest Coxeter group for which this definition is indeed more general than considering conjugates of a fixed simple

Received by the editors February 12, 2014 and, in revised form, July 9, 2014.

2010 Mathematics Subject Classification. Primary $20 \mathrm{~F} 55$.

The first and fourth authors were supported by the DFG through SFB 701 "Spectral Structures and Topological Methods in Mathematics".

The third author was supported by the DFG through STU 563/2-1 "Coxeter-Catalan combinatorics". 
system is the finite Coxeter group of type $H_{2}=I_{2}(5)$ given by all linear transformations of the plane that leave a regular pentagon invariant. One choice for a simple system is given by two reflections through two consecutive vertices of the pentagon, another choice is the product of two reflections through two vertices with distance two. Both choices of simple systems generate the same set of reflections, even though they are not conjugate.

The braid group on $n$ strands is the group $B_{n}$ with generators $\sigma_{1}, \ldots, \sigma_{n-1}$ subject to the relations

$$
\begin{aligned}
\sigma_{i} \sigma_{j} & =\sigma_{j} \sigma_{i} \text { for }|i-j|>1, \\
\sigma_{i} \sigma_{i+1} \sigma_{i} & =\sigma_{i+1} \sigma_{i} \sigma_{i+1} .
\end{aligned}
$$

It acts on the set $T^{n}$ of $n$-tuples of reflections as

$$
\begin{aligned}
\sigma_{i}\left(t_{1}, \ldots, t_{n}\right) & =\left(t_{1}, \ldots, t_{i-1}, t_{i} t_{i+1} t_{i}, \quad t_{i} \quad, t_{i+2}, \ldots, t_{n}\right), \\
\sigma_{i}^{-1}\left(t_{1}, \ldots, t_{n}\right) & =\left(t_{1}, \ldots, t_{i-1}, \quad t_{i+1}, t_{i+1} t_{i} t_{i+1}, t_{i+2}, \ldots, t_{n}\right) .
\end{aligned}
$$

For example, if $n=2$, then the action of $\sigma_{1}$ is described by

$$
\ldots \mapsto(\text { srs, srsrs }) \mapsto(s, s r s) \mapsto(r, s) \mapsto(\text { rsr }, r) \mapsto(\text { rsrsr }, r s r) \mapsto \ldots
$$

for any $r, s \in T$. Note that in this case, the $B_{2}$-orbit of $(r, s)$ is the set of all pairs $\left(t_{1}, t_{2}\right)$ of reflections of the subgroup $\langle r, s\rangle$, such that $t_{1} t_{2}=r s$.

The following lemma is a direct consequence of the definition.

Lemma 1.2. Let $W^{\prime}$ be a reflection subgroup of $W$ and let $T^{\prime}=T \cap W^{\prime}$ be the set of reflections in $W^{\prime}$. For an element $w \in W^{\prime}$ with reduced T-factorization $w=t_{1} \cdots t_{n}$, the braid group on $n$ strands acts on $\operatorname{Red}_{T^{\prime}}(w)$.

Proof. Let $\sigma_{i}\left(t_{1}, \ldots, t_{n}\right)=\left(t_{1}^{\prime}, \ldots, t_{n}^{\prime}\right)$. The lemma follows from the two observations that $t_{1} \cdots t_{n}=t_{1}^{\prime} \cdots t_{n}^{\prime}$ and $\left\{t_{1}, \ldots, t_{n}\right\} \subseteq T^{\prime}$ if and only if $\left\{t_{1}^{\prime}, \ldots, t_{n}^{\prime}\right\} \subseteq$ $T^{\prime}$.

This action on $\operatorname{Red}_{T^{\prime}}(w)$ is also known as the Hurwitz action. For finite Coxeter systems, the Hurwitz action was first shown to act transitively on $\operatorname{Red}_{T}(c)$ for a Coxeter element $c$ in a letter from P. Deligne to E. Looijenga Del74]. The first published proof is due to D. Bessis and can be found in Bes03. K. Igusa and R. Schiffler generalized this result to arbitrary Coxeter groups of finite rank; see [IS10, Theorem 1.4]. This transitivity has important applications in the theory of Artin groups, see Bes03, Dig06, and as well as in the representation theory of algebras; see [IS10, Igu11, HK13].

The aim of this note is to provide a simple proof of K. Igusa and R. Schiffler's theorem, based on arguments similar to those in Dye01. We moreover emphasize that the condition on the Coxeter element $c \in W$ in this note is slightly relaxed from the condition in the original theorem; compare [S10, Theorem 1.4].

Theorem 1.3. Let $(W, T)$ be a dual Coxeter system of finite rank $m$ and let $c=$ $s_{1} \cdots s_{n}$ be a parabolic Coxeter element in $W$. The Hurwitz action on $\operatorname{Red}_{T}(c)$ is transitive. In symbols, for each $\left(t_{1}, \ldots, t_{n}\right) \in T^{n}$ such that $c=t_{1} \cdots t_{n}$, there is a braid group element $b \in B_{n}$ such that

$$
b\left(t_{1}, \ldots, t_{n}\right)=\left(s_{1}, \ldots, s_{n}\right) .
$$


By the observation in Lemma 1.2, this theorem has the direct consequence that the parabolic subgroup $\left\langle s_{1}, \ldots, s_{n}\right\rangle$ of $W$ does indeed not depend on the particular $S$-factorization $c=s_{1} \cdots s_{n}$ but only on the parabolic Coxeter element $c$ itself. We thus denote this parabolic by $W_{c}:=\left\langle t_{1}, \ldots, t_{n}\right\rangle$ for any $T$-factorization $c=t_{1} \cdots t_{n}$. We moreover obtain that $\operatorname{Red}_{T}(c)=\operatorname{Red}_{T^{\prime}}(c)$ with $T^{\prime}=W_{c} \cap T$ being the set of reflections in the parabolic subgroup $W_{c}$. The main argument in the proof of this theorem (see Proposition 2.2 below) will also imply the following theorem that extends this direct consequence to all elements in a parabolic subgroup.

Theorem 1.4. Let $W^{\prime}$ be a parabolic subgroup of $W$. Then for any $w \in W^{\prime}$,

$$
\operatorname{Red}_{T}(w)=\operatorname{Red}_{T^{\prime}}(w),
$$

where $T^{\prime}=W^{\prime} \cap T$ is the set of reflections in $W^{\prime}$.

\section{THE PROOF}

For the proof of the two theorems, we fix a Coxeter system $(W, S)$. Denote by $\ell=$ $\ell_{S}$ and by $\ell_{T}$ the length function on $W$ with respect to the simple generators $S$ and with respect to the generating set $T$, respectively. Since $S \subseteq T$, we have that $\ell_{T}(w) \leq \ell(w)$ for all $w \in W$.

The following lemma provides an alternative description of standard parabolic Coxeter elements.

Lemma 2.1. An element $w \in W$ is a standard parabolic Coxeter element for $(W, S)$ if and only if $\ell_{T}(w)=\ell(w)$.

Proof. Given a reduced $S$-factorization $w=s_{i_{1}} \ldots s_{i_{k}}$, it was shown in Dye01, Theorem 1.1] that $\ell_{T}(w)$ is given by the minimal number of simple generators that can be removed from $s_{i_{1}} \ldots s_{i_{k}}$ to obtain the identity. This yields that $\ell_{T}(w)=$ $k=\ell(w)$ if and only if $s_{i_{1}} \ldots s_{i_{k}}$ does not contain any generator twice.

Define the Bruhat graph $\Omega$ for the dual Coxeter system $(W, T)$ as the undirected graph on vertex set $W$ with edges given by $w-w t$ for $t \in T$. For any factorization $w=t_{1} \cdots t_{n} \in W$ with $t_{i} \in T$ and any $x \in W$, there is a corresponding path

$$
x-x t_{1}-x t_{1} t_{2}-\ldots-x t_{1} \cdots t_{n}=x w
$$

from $x$ to $x w$ in $\Omega$. It is clear that this $T$-factorization of $w$ is reduced if and only if the corresponding path from $x$ to $x w$ has minimal length among paths from $x$ to $x w$ for some (equivalently, every) $x \in W$. The simple system $(W, S)$ induces an orientation on $\Omega$ given by $w \rightarrow w t$ if $\ell(w)<\ell(w t)$. We denote the resulting directed Bruhat graph by $\Omega_{\text {dir }}$.

The proof of the two main results is based on the case $x=e$ of the following proposition.

Proposition 2.2. Let $(W, S)$ be a Coxeter system. Moreover, let $w=t_{1} \cdots t_{n} \in W$ be a reduced $T$-factorization of an element in $W$, and let

$$
x-x t_{1}-x t_{1} t_{2}-\ldots-x t_{1} \cdots t_{n}=x w
$$

be the corresponding path in $\Omega$ starting at an element $x \in W$. Then there is a $T$ factorization $w=t_{1}^{\prime} \cdots t_{n}^{\prime}$ in the Hurwitz orbit of the T-factorization $t_{1} \cdots t_{n}$ such that the corresponding path in $\Omega_{\mathrm{dir}}$ starting at $x$ is first decreasing in length, then increasing; more precisely, it is of the form

$$
x \leftarrow x t_{1}^{\prime} \leftarrow x t_{1}^{\prime} t_{2}^{\prime} \leftarrow \ldots \leftarrow x t_{1}^{\prime} \cdots t_{i}^{\prime} \rightarrow \ldots \rightarrow x t_{1}^{\prime} \cdots t_{n}^{\prime}=x w
$$


for some (unique) integer $i$ with $0 \leq i \leq n$. In the special case $x=e$, this gives a directed path

in $\Omega_{\text {dir }}$.

$$
e \rightarrow t_{1}^{\prime} \rightarrow t_{1}^{\prime} t_{2}^{\prime} \rightarrow \ldots \rightarrow t_{1}^{\prime} \cdots t_{n}^{\prime}=w
$$

Proof. First consider two distinct reflections $t_{1}$ and $t_{2}$ and an element $z \in W$ such that $z \rightarrow z t_{1} \leftarrow z t_{1} t_{2}$ in $\Omega_{\mathrm{dir}}$. We claim that there exist reflections $t_{1}^{\prime}, t_{2}^{\prime} \in\left\langle t_{1}, t_{2}\right\rangle$ with $t_{1} t_{2}=t_{1}^{\prime} t_{2}^{\prime}$ such that $z \longrightarrow z t_{1}^{\prime} \rightarrow z t_{1}^{\prime} t_{2}^{\prime}$ or $z \leftarrow z t_{1}^{\prime} \leftarrow z t_{1}^{\prime} t_{2}^{\prime}$ or $z \leftarrow z t_{1}^{\prime} \rightarrow z t_{1}^{\prime} t_{2}^{\prime}$. This implies, by the comment before Lemma 1.2, that one can get from the factorization $t_{1} t_{2}$ to the factorization $t_{1}^{\prime} t_{2}^{\prime}$ inside $W^{\prime}=\left\langle t_{1}, t_{2}\right\rangle$ by braid moves, and hence in particular that $W^{\prime}=\left\langle t_{1}^{\prime}, t_{2}^{\prime}\right\rangle$. Moreover, one has $\ell\left(z t_{1}^{\prime}\right)<\max \left(\ell(z), \ell\left(z t_{1} t_{2}\right)\right)<$ $\ell\left(z t_{1}\right)$.

To prove the claim, consider the coset $z\left\langle t_{1}, t_{2}\right\rangle$ in $W$. By Dye01, Theorem 2.1], the proof immediately reduces to the case $z=e$ and $W^{\prime}=\left\langle t_{1}, t_{2}\right\rangle$ dihedral. We check this case directly. To this end, let $s_{1}^{\prime}, s_{2}^{\prime}$ be the Coxeter generators of $W^{\prime}$, and observe that any reflection (element of odd length) and any rotation (element of even length) in $W^{\prime}$ are joined by an edge in $\Omega$, which in $\Omega_{\text {dir }}$ is oriented towards the element of greater length with respect to the generating set $s_{1}^{\prime}, s_{2}^{\prime}$. Given $z \in W^{\prime}$, there are three situations: either $\ell(z)<\ell\left(z t_{1} t_{2}\right)$, or $\ell(z)>\ell\left(z t_{1} t_{2}\right)$, or $\ell(z)=\ell\left(z t_{1} t_{2}\right)$. This implies that one can choose $t_{1}^{\prime}$ and $t_{2}^{\prime}$ with $t_{1}^{\prime} t_{2}^{\prime}=t_{1} t_{2}$ in the three situations such that $z \rightarrow z t_{1}^{\prime} \rightarrow z t_{1}^{\prime} t_{2}^{\prime}, z \leftarrow z t_{1}^{\prime} \leftarrow z t_{1}^{\prime} t_{2}^{\prime}$ or $z \leftarrow z t_{1}^{\prime} \rightarrow z t_{1}^{\prime} t_{2}^{\prime}$ respectively (note that in the third case, one has $z \neq e$ or else $l\left(z t_{1} t_{2}\right)=l(z)$ implies $t_{1}=t_{2}$, contrary to assumption).

Consider the path in $\Omega$ attached to $w=t_{1} \cdots t_{n}$ and beginning at $x$. Any subpath $z-z t_{1}-z t_{1} t_{2}$ as in the claim may be replaced by a path $z-z t_{1}^{\prime}-z t_{1}^{\prime} t_{2}^{\prime}$ as there, to give a new path from $x$ to $x w$ of the same length $n$; we call this a "replacement." Apply to the original path a sequence of successive replacements. Any path so obtained corresponds to the path beginning at $x$ attached to some reduced $T$-factorization of $w$ in the same Hurwitz orbit as $t_{1} \cdots t_{n}$, and is a shortest path in $\Omega$ from $x$ to $x w$. Note that a replacement of any subpath $z \rightarrow z t_{1}^{\prime} \leftarrow z t_{1}^{\prime} t_{2}^{\prime}$ of such a path is possible since the path's minimal length implies that $t_{1}^{\prime} \neq t_{2}^{\prime}$. Each replacement decreases the total sum of the $\ell$-lengths of the vertices of the path, so eventually one obtains a path in which no further replacements are possible i.e. of the desired decreasing-then-increasing form. Finally, if $x=e$, then $i=0$ since there are no paths $e \leftarrow t$.

Given this proposition, we are finally in the position to prove the two main results of this note.

Proof of Theorem 1.4. Consider a Coxeter system $(W, S)$, a reflection subgroup $W^{\prime}$ of $W$. It is known that the directed Bruhat graph for $W^{\prime}$ corresponding to the simple system of $W^{\prime}$ induced by $S$ is the full subgraph $\left.\Omega_{\mathrm{dir}}\right|_{W^{\prime}}$ of $\Omega_{\mathrm{dir}}$ on vertex set $W^{\prime}$; see Dye01, Theorem 2.1].

Let $w \in W^{\prime}$. Then Lemma 1.2. Proposition 2.2 (with $x=e$ ), and the discussion before Proposition 2.2 imply that $\operatorname{Red}_{T}(w)=\operatorname{Red}_{T^{\prime}}(w)$ if and only if every shortest directed path from $e$ to $w$ in $\Omega_{\text {dir }}$ lies inside $\left.\Omega_{\text {dir }}\right|_{W^{\prime}}$.

Now assume that $W^{\prime}$ is a standard parabolic subgroup generated by some subset of $S$. Then it is well known that every reduced $S$-factorization for $w \in W^{\prime}$ is actually inside $W^{\prime}$. (To see this, recall that Coxeter systems have the property that all reduced $S$-factorizations for $w$ are related by braid relations.) It therefore 
follows that in this situation any shortest directed path from $e$ to $w$ in $\Omega_{\text {dir }}$ indeed lies inside $\left.\Omega_{\mathrm{dir}}\right|_{W^{\prime}}$. The theorem follows by the above equivalence.

Proof of Theorem 1.3. Again, fix a parabolic Coxeter element $c=s_{1} \cdots s_{n} \in W$ and a corresponding simple system $(W, S)$, and denote by $\Omega$ and $\Omega_{\text {dir }}$ the undirected and directed version of the Bruhat graph for $(W, S)$. By Proposition 2.2, it is left to show that any two directed paths from $e$ to $c$ in $\Omega_{\text {dir }}$ are in the same Hurwitz orbit. Let therefore

$$
e \rightarrow t_{1} \rightarrow t_{1} t_{2} \rightarrow \ldots \rightarrow t_{1} \cdots t_{n}=c
$$

be such a path. We have seen in Lemma 2.1 that $\ell(c)=\ell_{T}(c)=n$. It thus follows that $\ell\left(t_{1} \cdots t_{i}\right)=\ell_{T}\left(t_{1} \cdots t_{i}\right)=i$ for any $i$. The strong exchange condition, see e.g. Hum90, Theorem 5.8], then yields that $t_{1} \cdots t_{i+1}$ is obtained from $t_{1} \cdots t_{i}$ by adding a single simple generator into its position within $s_{1} \cdots s_{n}$. Therefore, such a path is (bijectively) encoded by a permutation $\pi=\left[\pi_{1}, \ldots, \pi_{n}\right]$ where $\pi_{i}$ is the index of the simple generator added at the $i$-th step. Given the factorization corresponding to such a path, it is straightforward to see that the embedding of the permutation into the braid group (by sending a simple transposition $(i, i+1)$ to the generator $\sigma_{i}$ of $B_{n}$ ) yields a braid that turns the given factorization into the factorization $s_{1} \cdots s_{n}$. To this end, observe that given two factorizations encoded by two permutations $\pi_{1}$ and $\pi_{2}$ with $\ell\left(\pi_{1}\right)=\ell\left(\pi_{2}\right)+1$ and such that these differ only by a single simple transposition $\pi_{2}^{-1} \pi_{1}=(i, i+1)$ for some index $i$. Then the given factorizations are obtained from each other by applying the braid group generator $\sigma_{i}$ to the factorization corresponding to $\pi_{1}$ to obtain the factorization corresponding to $\pi_{2}$. As the factorization corresponding to the identity permutation $[1, \ldots, n]$ is $\left(s_{1}, \ldots, s_{n}\right)$, the claim follows.

As an example of the construction in the previous proof, consider the path

$$
e \rightarrow s_{2} \rightarrow s_{2} s_{5} \rightarrow s_{2} s_{3} s_{5} \rightarrow s_{1} s_{2} s_{3} s_{5} \rightarrow s_{1} s_{2} s_{3} s_{4} s_{5}=c .
$$

The corresponding factorization of $c$ is given by

$$
c=s_{2} \cdot s_{5} \cdot s_{5} s_{3} s_{5} \cdot s_{5} s_{3} s_{2} s_{1} s_{2} s_{3} s_{5} \cdot s_{5} s_{4} s_{5},
$$

and the permutation is $\pi=[2,5,3,1,4]=(1,2)(2,3)(4,5)(3,4)(2,3)$. On the other hand,

$$
\sigma_{1} \sigma_{2} \sigma_{4} \sigma_{3} \sigma_{2}\left(s_{2}, s_{5}, s_{5} s_{3} s_{5}, s_{5} s_{3} s_{2} s_{1} s_{2} s_{3} s_{5}, s_{5} s_{4} s_{5}\right)=\left(s_{1}, s_{2}, s_{3}, s_{4}, s_{5}\right),
$$

as desired.

\section{REFERENCES}

[Bes03] David Bessis, The dual braid monoid (English, with English and French summaries), Ann. Sci. École Norm. Sup. (4) 36 (2003), no. 5, 647-683, DOI 10.1016/j.ansens.2003.01.001. MR2032983(2004m:20071)

[Del74] P. Deligne, letter to E. Looijenga, 9/3/1974. available at http://homepage.univie.ac. at/christian.stump/Deligne_Looijenga_Letter_09-03-1974.pdf .

[Dig06] F. Digne, Présentations duales des groupes de tresses de type affine $\widetilde{A}$ (French, with English summary), Comment. Math. Helv. 81 (2006), no. 1, 23-47, DOI 10.4171/CMH/41. MR2208796 (2006k:20075)

[Dye01] Matthew J. Dyer, On minimal lengths of expressions of Coxeter group elements as products of reflections, Proc. Amer. Math. Soc. 129 (2001), no. 9, 2591-2595 (electronic), DOI 10.1090/S0002-9939-01-05876-2. MR1838781(2002e:20075) 
[Dye90] Matthew Dyer, Reflection subgroups of Coxeter systems, J. Algebra 135 (1990), no. 1, 57-73, DOI 10.1016/0021-8693(90)90149-I. MR1076077 (91j:20100)

[Hum90] James E. Humphreys, Reflection groups and Coxeter groups, Cambridge Studies in Advanced Mathematics, vol. 29, Cambridge University Press, Cambridge, 1990. MR:1066460(92h:20002)

[Igu11] Kiyoshi Igusa, Exceptional sequences, braid groups and clusters, Groups, algebras and applications, Contemp. Math., vol. 537, Amer. Math. Soc., Providence, RI, 2011, pp. 227-240, DOI 10.1090/conm/537/10578. MR2799103(2012f:16001)

[IS10] Kiyoshi Igusa and Ralf Schiffler, Exceptional sequences and clusters, J. Algebra 323 (2010), no. 8, 2183-2202, DOI 10.1016/j.jalgebra.2010.02.003. MR.2596373 (2011b:20118)

[HK13] A. Hubery, H. Krause, A categorification of non-crossing partitions, arXiv:1310.1907 (2013), 30 pages

[FHM06] W. N. Franzsen, R. B. Howlett, and B. Mühlherr, Reflections in abstract Coxeter groups, Comment. Math. Helv. 81 (2006), no. 3, 665-697, DOI 10.4171/CMH/69. MR.2250859 (2007e:20080)

Fakultät für Mathematik, Universität Bielefeld, 33501 Bielefeld, Germany

E-mail address: b.baumeister@math.uni-bielefeld.de

Department of Mathematics, University of Notre Dame, 255 Hurley, Notre Dame, INDIANA 46556

E-mail address: matthew.j.dyer.1@nd.edu

Institut für Mathematik, Freie Universität Berlin, 14195 Berlin, Germany

E-mail address: christian.stump@fu-berlin.de

Fakultät für Mathematik, Universität Bielefeld, 33501 Bielefeld, Germany

E-mail address: patrick.wegener@math.uni-bielefeld.de 